\title{
Evaluation of the System of Dispensation of Drugs in Intrahospital Services, of a Health Unit of Riobamba
}

\section{Evaluación al Sistema de Dispensación de Medicamentos en los Servicios Intrahospita- larios, de una Unidad de Salud de Riobamba}

VII International Congress of Science, Technology, Entrepreneurship and Innovation (SECTEI 2020)

Corresponding Author:

A Rincón

adriana.rincon@espoch.edu.ec

Published: 26 August 2021

Production and Hosting by

Knowledge $E$

(c) V Rodríguez et al. This article is distributed under the terms of the Creative Commons Attribution License, which permits unrestricted use and redistribution provided that the original author and source are credited.

\section{Rodríguez ${ }^{1}, T$ Peñafiel ${ }^{2}$, and A Rincón ${ }^{1}$}

${ }^{1}$ Grupo de Investigación de Tecnología y Atención Farmacéutica, Facultad de Ciencias, Escuela Superior Politécnica de Chimborazo, Riobamba, Ecuador

${ }^{2}$ Botica Bristol Dirección, Larrea 22-34 y $1^{\circ}$ Constituyente, Frente al Parque Sucre, Riobamba, Ecuador

\section{Abstract}

Introduction: In Ecuador, many health institutions have not yet migrated to the Unit Dose Dispensation / Distribution System for Medications, which ensures the correct, safe and rational use of medications. Objective: The medication dispensing process in a Riobamba health unit was evaluated as part of the service quality improvement process. Methodology: Information was collected during three months of investigation, from the in-hospital services of hospitalization, intensive care unit, operating room and emergency. The instruments used for the evaluation corresponded to medical prescriptions, medication and medical device request sheets, medication return record sheets and labeling of boxes. Results: In the medical prescriptions as in the record sheets for the return of evaluated drugs, the main drawback was presented in the writing of the pharmaceutical form and the absence of mandatory data for the identification of the patient. In the total of the registers for the return of medications, the absence of the reason for the return of the medications was identified. The medication dispensing process was considered a critical point within the evaluation, since throughout the review period it was observed that the boxes did not have the minimum required labeling, for the correct identification of the patient. Conclusion: It was concluded that the dispensing system presented critical flaws, such as: deficiencies in the supply of medications, dispensing errors, lack of pharmacotherapeutic follow-up and little participation of the pharmaceutical professional.

Keywords: dispensing, medication, medication error.

\section{Resumen}

Introducción: En Ecuador, muchas instituciones de salud aún no migran al Sistema de Dispensación/Distribución de Medicamentos por Dosis Unitaria, el cual asegura la correcta, segura y racional utilización de los medicamentos. Objetivo: Se evaluó el proceso de dispensación de medicamentos en una unidad de salud de Riobamba, como parte del proceso de mejoramiento en la calidad del servicio. Metodología: Se recolectó información durante tres meses de investigación, de los servicios de intrahospitalarios de hospitalización, unidad de cuidados intensivos, quirófano y emergencia. Los instrumentos utilizados para la evaluación correspondieron a recetas médicas, hojas de requerimiento de medicamentos y dispositivos 
médicos, hojas de registro de devolución de medicación y rotulación de cajetines. Resultados: En las recetas médicas como en las hojas de registro de devolución de medicamentos evaluadas, el principal inconveniente se presentó en la escritura de la forma farmacéutica y la ausencia de datos obligatorios para identificación del paciente. En el total de los registros de devolución de medicamentos, se identificó la inexistencia del motivo de la devolución de los medicamentos. El proceso de dispensación de medicación, se consideró un punto crítico dentro de la evaluación, ya que durante todo el período de revisión se observó que los cajetines no contaban con la rotulación mínima requerida, para la identificación correcta del paciente. Conclusión: Se concluyó que el sistema de dispensación presentaba fallas críticas, tales como: deficiencias en el suministro de medicamentos, errores de dispensación, falta de seguimiento farmacoterapéutico y la poca participación del profesional farmacéutico.

Palabras Clave: dispensación, medicamentos, error de medicación.

\section{Introducción}

Los sistemas de salud, a través de los servicios de farmacia, deben asegurar la correcta, segura y efectiva utilización de los medicamentos en los hospitales, por lo que resulta indispensable mejorar la forma de dispensarlos por medio de la adherencia a las normativas establecidas por la Organización Mundial de la Salud y otros organismos regulatorios como la Alianza Mundial para la Seguridad del Paciente que tiene por objeto promover la investigación y facilitar la utilización de los resultados de la investigación en pro de una atención sanitaria más segura y una reducción del riesgo de ocasionar daño a los pacientes [1]. Los eventos adversos que pueden producirse debido a una atención poco segura son probablemente una de las principales causas de mortalidad o discapacidad a nivel mundial [2]. En los países desarrollados, se estima que uno de cada 10 pacientes puede sufrir daños durante su estancia intrahospitalaria [3]. Casi el 50\% de los daños que se producen son prevenibles, siendo los errores más perjudiciales los relacionados con el diagnóstico, la prescripción y el uso de medicamentos [4].

Desde la década de los años 60 , se asoció el alto grado de errores de medicación generado en los hospitales con el sistema de distribución de medicamentos empleado [5]; surgiendo como solución efectiva para disminuir los errores existentes en la prescripción, preparación y administración de medicamentos el Sistema de Dispensación de Medicamentos por Dosis Unitaria (SDMDU), el cual en el año 2001, constituía una realidad en aproximadamente el $90 \%$ de los hospitales en Estados Unidos y en España, formaba parte de los servicios que ofrecían más del $50 \%$ de las farmacia de hospital, por lo que, este sistema con más de 50 años de su aparición, ha sido elemento fundamental para la obtención de una farmacoterapia eficiente y segura [6]. En Ecuador, en el año 
2012 el Ministerio de Salud Pública estableció la 'Norma para la Aplicación del Sistema de Dispensación/Distribución de Medicamentos por Dosis Unitaria' para los hospitales del sistema nacional de salud, con el fin de permitir un mejoramiento continuo de los procesos ejecutados en el servicio de farmacia hospitalaria con respecto al ciclo del medicamento y el uso racional de los mismos [7], sin embargo, en muchas unidades de salud de índole privado, a pesar de los esfuerzos por implementar este modelo, aún se emplean procedimientos de dispensación clásicos. Los métodos tradicionales utilizados en la dispensación de medicamentos se centraban en el sistema de stock en planta, fundamentado en la disponibilidad de la medicación del paciente en la unidad de enfermería [8] y al sistema de petición por paciente, en el que el personal de enfermería solicitaba la medicación en forma individualizada al servicio de farmacia, que se dispensaba en forma global [9]

En cualquiera de los casos, el farmacéutico no tenía acceso directo a la orden médica original, ni participación en el equipo de salud, existiendo mayor riesgo de interpretación de la orden médica, errores de transcripción y, por tanto, menor control sobre la administración de medicamentos [10]. Es posible conocer si la población tiene acceso a los medicamentos esenciales, si realmente se realiza una adecuada prescripción y dispensación de los medicamentos, garantizando seguridad, eficacia y calidad. Resulta importante e indispensable, que las personas que reciben los medicamentos los empleen de una manera segura, constituyendo el farmacéutico una pieza clave en este proceso, ya que interviene de forma directa en la farmacoterapia de los pacientes, y es partícipe en el desarrollo de los protocolos terapéuticos [11].

Por lo anterior, el sistema de dispensación por dosis unitaria constituye irremediablemente la alternativa al sistema de distribución tradicional, garantizando la adecuada dispensación de medicamentos y el uso racional de los mismos [12], sin embargo, en las unidades de salud que aún no logran migrar en forma definitiva al SDMDU, es fundamental disminuir los errores al momento de la dispensación de medicamentos en las distintas áreas; puesto que, existen fallas como fuga de medicamentos, ejecución incorrecta de los reingresos de medicación, medicamentos no solicitados o solicitados incorrectamente en recetas, entre otros inconvenientes; que involucran a todo el personal de salud de los diferentes servicios hospitalarios [13]. Es así como, ante la posibilidad de errores, el objetivo de este estudio fue evaluar el proceso de dispensación de medicamentos en una unidad de salud de Riobamba, Ecuador, a fin de logar la detección de errores, como parte de un proceso de mejoramiento en la calidad del servicio, pudiendo en un plazo de tiempo determinado, migrar al SDMDU. 


\section{Materiales y Métodos}

Los servicios de Farmacia de las unidades operativas de salud del país, constituyen un eje fundamental para el buen desenvolvimiento en la prestación universal de los servicios de atención integral de salud, por lo que resulta imprescindible en todo momento garantizar la provisión y el uso seguro de los medicamentos, sin embargo, en muchas instituciones a nivel nacional aún no se cumplen con las normativas establecidas, siendo una de las causas principales, la falta de profesionales Farmacéuticos dedicados a realizar gestión e intervenciones farmacéuticas enfocados al paciente. A través de un proyecto de investigación propuesto se pretende acercar a todas las Unidades de Salud que sea posible, con el fin de identificar y analizar las necesidades que tengan sus servicios de Farmacia y de esta forma contribuir a la mejora continua de los mismos, por medio de la ejecución de estrategias que permitan gestionar, reestructurar e implementar las fortalezas necesarias para hacer de la Atención Farmacéutica y la Farmacia Hospitalaria un servicio de calidad. En tal sentido en una de las instituciones de salud de la ciudad de Riobamba, previo a una carta de cooperación interinstitucional se evaluó el proceso de dispensación de medicamentos que se realiza desde el área de farmacia hacia los servicios intrahospitalarios, teniendo en consideración la inexistencia del sistema de dosis unitaria en la unidad de salud. Para tal fin, fueron analizados los elementos indispensables e inherentes a la dispensación en cada uno de los servicios objeto de estudio, que a saber fueron: hospitalización, unidad de cuidados intensivos (UCI), quirófano y emergencia. Los instrumentos utilizados para la recolección de datos y el levantamiento de la información fueron evaluados, durante un periodo de tres meses de investigación y provinieron del servicio de farmacia; así como de los servicios intrahospitalarios anteriormente mencionados. Los parámetros que se constataron en cada los instrumentos evaluados, se detallan en la Tabla 1.

\section{Resultados y Discusión}

Fueron evaluadas 890 recetas médicas que llegaron al servicio de farmacia desde los servicios intrahospitalarios, en las cuales se evidenció que en un $70 \%$ de las recetas, no existían datos informativos como nombre o cédula de identidad o datos obligatorios para identificación del paciente. Otro parámetro informativo que fue omitido correspondió al diagnóstico, a través de su equivalente, la clasificación internacional de las enfermedades (CIE 10), debiéndose este problema posiblemente a la falta de conocimiento por parte del personal, acerca de la codificación de las enfermedades [14].

Tanto en las recetas médicas como en las hojas de registro de requerimientos de medicamentos, el principal inconveniente se presentó en la escritura de la forma 


\section{Table 1}

Instrumentos empleados para la evaluación del proceso de dispensación.

Instrumento evaluado
Recetas médicas
Hojas de requerimiento de
medicamentos y dispositivos
médicos
Hojas de registro de devolución
de medicación (Hojas de costos)
Rotulación de cajetines

Servicios generadores del
instrumento
-Hospitalización
- Unidad de cuidados intensivos
-Emergencia
-Emergencia
-Quirófano
-Hospitalización
-Servicio de farmacia para
servicio de hospitalización

Parámetros de verificación
-Datos del paciente
- Datos del medicamento
-Datos del prescriptor
-Solicitud de medicamentos
dispositivos médicos e insumos
-Devolución final de los
medicamentos sobrantes
-Sala
- Nombre del paciente
- Cédula de identidad
-Número de historia clínica
-Número de cama

farmacéutica de los medicamentos, tanto sólidos como líquidos, según lo indicado por el Ministerio de Salud Pública. Este hallazgo fue detectado en el $100 \%$ del total de formatos evaluados. Con respecto, a este aspecto es importante destacar que la prescripción en formato manual de un tratamiento farmacológico puede ser considerada como un punto crítico para la seguridad de los pacientes, puesto que las indicaciones del médico tratante descritas en la historia clínica, generalmente son transcritas por el residente o personal médico de apoyo [15].

De esta forma, también se encontró que la cantidad de medicamentos solicitados en la receta médica fue diferente a la prescrita, siendo en la mayoría de los casos superior a la descrita en la historia clínica del paciente, situación que ocurrió de manera particular en el servicio de Unidad de Cuidados Intensivos ( $\mathrm{UCl}$ ), donde este error se evidenció en el $73,7 \%$, de un total de 38 recetas médicas evaluadas en este servicio.

Los errores de medicación, en forma farmacéutica y cantidad pueden constituir errores determinantes en la terapia farmacológica del paciente, ocasionando en el caso más grave nuevas enfermedades o problemas relacionados con los medicamentos (PRMs) [16]. Algunos estudios mencionan que cada año mueren entre $44000 \mathrm{y}$ 98000 personas a causa de errores de medicación, indicando que la morbimortalidad producidas por los medicamentos son muy elevadas. De igual forma estos errores, además del costo en vidas humanas, pueden generar costos significativos en términos monetarios, pérdida de confianza por parte de los pacientes en el sistema de salud y disminución en la satisfacción de los pacientes y los profesionales [17]

Con respecto a los formatos de devolución de medicamentos, fueron evaluados 58 , los cuales eran provenientes del área de hospitalización y que se manejaban sólo para la devolución final de los medicamentos sobrantes, a fin de controlar el costo de estos. El proceso de devolución era realizado por el personal de enfermería que 
entregaba al servicio de farmacia, la medicación junto con el formato de devolución, en los cuales se observó como error más frecuente la omisión de la forma farmacéutica del medicamento (98\%). Además, en todos los formatos evaluados, no se registraban datos fundamentales como, motivo de la devolución de los medicamentos, fecha de caducidad, lote del medicamento y firma del responsable. Por último, se evidenció que la devolución de los medicamentos no se realizaba por día, por lo que a farmacia llegaban acumulados y en ocasiones de varios servicios, lo cual generaba inconvenientes importantes como, riesgo de confusión, existencia de desviaciones en la planeación estratégica de compras y adquisiciones, proyección de inventarios, pérdidas por vencimiento y desajustes en la estimación de necesidades para la unidad de salud [18].

Finalmente, se evaluó el proceso de entrega de medicación desde el servicio de farmacia, el cual se efectuaba través del coche de medicación, determinándose como un punto crítico dentro de la evaluación del proceso de dispensación, ya que durante todo el período de revisión se observó que los cajetines no contaban con la rotulación mínima requerida para una identificación correcta del paciente, existiendo únicamente en la rotulación, la sala y el número de cama donde se encontraba el paciente. Este resultado es de vital importancia, al considerar que el no realizar una correcta identificación de los cajetines o bolsas de medicación conlleva errores que pueden alcanzar al paciente y provocar daños en ellos, ya que muchas de las veces la rotación del personal implica, que no se tenga la información adecuada de cada paciente, siendo así frecuente estos errores cuando existe cambio de cama, suspensión de un medicamento, cambio de vía o cuando simplemente el personal de enfermería o auxiliar, no puede verificar que sea el mismo paciente al que se le va administrar la medicación [19].

Para mejorar la calidad de vida del paciente no basta que el medicamento sea producido con calidad; es necesaria la intervención del profesional farmacéutico a lo largo de toda la trayectoria del medicamento hasta lograr el objetivo terapéutico para el que fue indicado, por ello es imprescindible la existencia de medidas que verifiquen una correcta y eficaz dispensación de medicamentos y que propicien su utilización racional, a través de un sistema idóneo para tal fin [20]. Resultados que se detallan en la Tabla 2.

\section{Conclusiones}

La dispensación de medicamentos en la unidad de salud evaluada se ejecutaba por los auxiliares de farmacia, a través de un sistema tradicional de entrega y despacho de medicación, no participando en forma directa el profesional químico o bioquímico farmacéutico y no existiendo un Sistema de Dispensación de Medicamentos por Dosis 
Table 2

Errores encontrados en los instrumentos evaluados provenientes de los servicios intrahospitalarios de la unidad de salud.

\begin{tabular}{|c|c|c|c|c|c|c|c|c|c|c|}
\hline \multirow[t]{2}{*}{ Errores } & \multicolumn{2}{|c|}{$\begin{array}{l}\text { Hospitalización } \\
\text { /Paliativos }\end{array}$} & \multicolumn{2}{|c|}{$\mathrm{UCl}$} & \multicolumn{2}{|c|}{ Endoscopía } & \multicolumn{2}{|c|}{ Emergencia } & \multirow{2}{*}{$\begin{array}{l}\text { Total } \\
\text { No. }\end{array}$} & \multirow{2}{*}{$\begin{array}{l}\% \\
\%\end{array}$} \\
\hline & No. & $\%$. & No. & $\%$ & No. & $\%$ & No. & $\%$ & & \\
\hline $\begin{array}{l}\text { Nombres y Apellidos } \\
\text { completos }\end{array}$ & 0 & 0 & 5 & 13,2 & 3 & 4,8 & 30 & 33,7 & 38 & 4.3 \\
\hline $\begin{array}{c}\text { Cédula de ident idad o } \\
\text { pasaporte para } \\
\text { personas extr ajeras }\end{array}$ & 700 & 100 & 38 & 100 & 63 & 100 & 89 & 100 & 890 & 100 \\
\hline $\begin{array}{c}\text { Número de historia } \\
\text { clínica }\end{array}$ & 43 & 6,1 & 0 & 0 & $o^{n}$ & $\begin{array}{r}\text { no } \\
\text { aplica }\end{array}$ & $o^{n}$ & $\begin{array}{c}\mathrm{n} \\
\mathrm{o}^{\mathrm{aplica}}\end{array}$ & 43 & 4.8 \\
\hline Edad en años y meses & 0 & 0 & 0 & 0 & $\begin{array}{l}\text { no se } \\
\text { usa }\end{array}$ & $\begin{array}{c}\text { no se } \\
\text { usa }\end{array}$ & $\begin{array}{c}\text { no se } \\
\text { usa }\end{array}$ & $\begin{array}{r}\text { no se } \\
\text { usa }\end{array}$ & $u$ & u \\
\hline CIE 10 & 700 & 100 & 38 & 100 & 63 & 100 & 89 & 100 & 890 & 100 \\
\hline $\begin{array}{l}\text { Sexo masculino o } \\
\text { femenino }\end{array}$ & 700 & 100 & 38 & 100 & 63 & 100 & 89 & 100 & 890 & 100 \\
\hline Nacionalidad & 700 & 100 & 38 & 100 & 63 & 100 & 89 & 100 & 890 & 100 \\
\hline Numero de cama & 15 & $1, y$ & $\angle 0$ & 6ర,4 & $\begin{array}{r}\text { no se } \\
\text { usa }\end{array}$ & $\begin{array}{l}\text { no se } \\
\text { usa }\end{array}$ & $\begin{array}{l}\text { no se } \\
\text { usa }\end{array}$ & $\begin{array}{r}\text { no se } \\
\text { usa }\end{array}$ & sy & 4.4 \\
\hline $\begin{array}{c}\text { Nombre genérico o } \\
\text { DCI }\end{array}$ & 0 & 0 & 0 & 0 & 0 & 0 & 0 & 0 & 0 & 0 \\
\hline Concentración & 0 & 0 & 0 & 0 & 0 & 0 & 0 & 0 & 0 & 0 \\
\hline Forma farmacéutica & 700 & 100 & 38 & 100 & 63 & 100 & 89 & 100 & 890 & 100 \\
\hline $\begin{array}{c}\text { Cantidad en números y } \\
\text { letras }\end{array}$ & 36 & 5,1 & 28 & 73,7 & 3 & 4,8 & 7 & 7,9 & 74 & 8.3 \\
\hline $\begin{array}{l}\text { Nombre y apellido del } \\
\text { médico }\end{array}$ & 53 & 7,6 & 38 & 100 & 56 & 88,9 & 89 & 100 & 236 & 26.5 \\
\hline $\begin{array}{l}\text { Firma y sello de } \\
\text { facultativo }\end{array}$ & 4 & 0,6 & 0 & 0 & 0 & 0 & 0 & 0 & 4 & 0.4 \\
\hline $\begin{array}{l}\text { Número total de } \\
\text { recetas evaluadas }\end{array}$ & 700 & 100 & 38 & 100 & 63 & 100 & 89 & 100 & 890 & 100 \\
\hline
\end{tabular}

Unitaria, encontrándose importantes errores de medicación que con mayor frecuencia correspondieron a datos faltantes del paciente y $\mathrm{CIE}$ 10, descripción de las formas farmacéuticas y omisión de medicación en las recetas; evidenciándose la mayoría de estos errores en el área de hospitalización y UCl. El proceso de devolución de medicamentos al realizarse en forma inadecuada constituía una fuente de riesgo de confusión, existencia de desviaciones en la planeación estratégica de compras y adquisiciones, entre otros inconvenientes. La dispensación de medicamentos realizada a través de coche de medicación era objeto de riesgo en la dispensación al no contar con la rotulación adecuada de sus cajetines, lo que impedía la identificación del paciente. La evaluación realizada indica la necesidad inminente de migrar hacía un sistema de dispensación de medicamentos con la participación activa del equipo de salud que reduzca significativamente los errores de medicación y por tanto asegure seguridad, eficacia y uso racional de medicamentos. 


\section{Conflicto de Intereses}

Los autores declaran no tener conflicto de intereses.

\section{References}

[1] Organización Mundial de la Salud. Seguridad del paciente - Medidas mundiales en materia de seguridad del paciente. Informe del Director General; 2019.

[2] Jha AK. Patient safety - a grand challenge for healthcare professionals and policymakers alike. Paper presented at: Roundtable at the Grand Challenges Meeting of the Bill \& Melinda Gates Foundation; 2018

[3] Slawomirski L, Auraaen A, Klazinga N. The economics of patient safety: Strengthening a value-based approach to reducing patient harm at national level. Paris: OECD; 2017.

[4] Slawomirski L, Auraaen A, Klazinga N. The economics of patient safety in primary and ambulatory care: Flying blind. Paris: OECD; 2018.

[5] Merino-Bravo M. Evaluación de la implementación de una estrategia basada en Dosis Unitaria para el uso racional de medicamentos en los hospitales del Ministerio de Salud Pública del Ecuador en el periodo 2014-2016 [Postgraduate Thesis]. Quito: USFQ; 2017.

[6] Hernández M, Poveda J. Sistemas automáticos de dispensación de medicamentos. Barcelona: Combino Pharm; 2001

[7] Ministerio de la Salud Pública. Norma para la aplicación del sistema de dispensación/distribución de medicamentos por dosis unitaria en los hospitales del sistema nacional de salud. 2012

[8] Zavala-González, Antonio M, et al. Efectividad de las intervenciones para mejorar la prescripción de medicamentos en atención primaria. Atención Primaria. 2017;1:13-20.

[9] Catalá P, González S. Portal Sociedad Española de Farmacia Hospitalaria Madrid. 2020.

[10] Organización Panamericana de la Salud. Servicios farmacéuticos basados en la atención primaria de salud. Documento de posición de la OPS-/OMS. 2013.

[11] Mercado E, Arteaga M. Viabilidad de un servicio farmacéutico en el centro de salud del corregimiento de Zungo Embarcadero (Carepa: zona bananera de Urabá). 2014.

[12] Napal V, González J, Ferrándiz R. Dispensación con intervención previa del Farmacéutico: Dosis unitarias. Farmacia Hospitalaria.

[13] Alfaro A, Quirós A, Rocha M. Errores detectados en la dispensación de medicamentos en un hospital público en Costa Rica. Pharmaceutical Care La Farmacoterapia. 2021;1:11-16. 
[14] Herrera LJ. El rol de farmacia en la vigilancia de la salud en Costa Rica. Revista Cubana de Salud Pública. 2016:418-431.

[15] Kern Pharma. Los farmacéuticos, ayer y hoy. Kern Pharma; 2017.

[16] De Andrés L, María A. Acerca de los errores de medicación en los servicios de urgencias hospitalarios: Pasos para la mejora en la seguridad del paciente. Emergencias. 2017; 6.

[17] Contreras PE, Galán MAR. Errores de medicación. MSPCH; 2016.

[18] Morimitsu T, Pablo J, Giraldo RDZ, Casas LAA. Plan de mejoramiento al proceso interno de las devoluciones de medicamentos y dispositivos médicos entre el servicio farmacéutico y los servicios hospitalarios de la ESE Hospital Universitario San Jorge de Pereira [Dissertation]. Universidad Libre de Pereira; 2015.

[19] Cancino FIG, Vega EM. Medicamentos de venta bajo receta médica retenida: Caracterización y análisis desde la dispensación. Rev Colomb Cienc Quím-Farm. 2014;2:272-83.

[20] Odalis RG, Julia GMA, Liuba AC, Pablo LC. La dispensación como herramienta para lograr el uso adecuado de los medicamentos en atención primaria. Rev Cubana Med Gen Integr. 2017;33(4).373 